Cómo citar este trabajo: Contreras Castellano, Carmen y Lucas Matheu, Manuel (2021). Experiencias de mujeres que han sido diagnosticadas de vaginismo. Una aproximación sociológica cualitativa. Revista del Laboratorio Iberoamericano para el Estudio Sociohistórico de las Sexualidades, 5, art. 4, pp. 80-98 https://doi.org/10.46661/ relies.5295

\title{
Experiencias de mujeres que han sido diagnosticadas de vaginismo. Una aproximación sociológica cualitativa
}

\author{
Experiences of women who have been diagnosed os vaginismus. A \\ qualitative sociological approach
}

\author{
Carmen Contreras Castellano \\ Universidad de Almería \\ Carmen.nerva.1995@gmail.com \\ [https://orcid.org/0000-0002-6834-0162] \\ Manuel Lucas Matheu \\ Universidad de Almería \\ seddepiel@gmail.com
}

[https://orcid.org/0000-0002-1551-5854]

\section{Resumen}

El vaginismo es una dificultad sexual femenina que se caracteriza por la imposibilidad de practicar el coito o la penetración vaginal. Basado en la escasez de estudios sobre vaginismo desde una perspectiva sociocultural, el objetivo de esta investigación fue explorar las experiencias subjetivas de mujeres heterosexuales con vaginismo, mediante una aproximación sociológica, a través de una metodología cualitativa. El estudio se realizó a partir de entrevistas en profundidad como técnica de recopilación de datos. Los resultados obtenidos a través de un análisis temático encontraron que los discursos, encauzados en sus vidas afectivas y sexuales, están marcados por la percepción de sentirse disfuncionales e influidos por un modelo normativo de la sexualidad, caracterizado por ser coitocéntrico y genitalizado. Finalmente, el artículo concluye con la necesidad de un abordaje profesional dirigido a despatologizar el vaginismo y a deconstruir los discursos normativos de la sexualidad, permitiendo que el coito deje de considerarse esencial y priorizando prácticas alternativas de placer. 
Palabras clave: vaginismo, coitocentrismo, sexualidad, tratamiento.

\begin{abstract}
Vaginismus is a female sexual difficulty characterized by the inability to carry out intercourse or vaginal penetration. Based on the scarcity of studies on vaginismus from a sociocultural perspective, the objective of this research was to explore the subjective experiences of women with vaginismus, through a sociological approach through a qualitative methodology. The study was conducted through in-depth interviews with women who had been diagnosed with vaginismus, as a data collection technique. The results obtained through a thematic analysis found that the speeches, channeled in their affective and sexual lives, are marked by the perception of feeling dysfunctional and influenced by a normative model of sexuality, characterized by being coitocentric and genitalized. Finally, the article concludes with the need for a professional approach aimed at depathologizing vaginismus and deconstructing the normative discourses of sexuality, allowing intercourse to cease to be considered essential and prioritizing alternative pleasure practices.
\end{abstract}

Key words: vaginismus; coitocentrism; sexuality; treatment. 


\section{Introducción}

Las disfunciones sexuales son percibidas como variaciones o alteraciones dentro de alguna de las fases de la respuesta sexual. En 1980 aparecieron por primera vez en una nueva sección del Manual Diagnóstico y Estadístico de los Trastornos Mentales (DSM III), de la Asociación Psiquiátrica Americana, obteniendo carácter clínico y asumiendo la existencia de tratamientos específicos para su supuesta resolución (Sánchez et al., 2010.).

La quinta y última edición del Manual Diagnóstico y Estadístico de los Trastornos Mentales (DSMV), identifica el vaginismo como un trastorno genito-pélvico de dolor/penetración (GPPPD), conceptualizándolo como disfunción sexual femenina (American Psychiatric Association, 2013).

El vaginismo principalmente es una dificultad sexual que impide el coito o la penetración vaginal y suele venir acompañado de dolor (Pacik, et al, 2019). Se desconoce la etiología exacta del vaginismo, asimismo, se ignora su verdadera prevalencia debido al silencio que gira en torno a esta dificultad sexual, aunque se estima que afecta alrededor del 5 al 17\% de las mujeres en un entorno clínico (Moltedo-Perfetti et al., 2014, Pacik, 2014; Maseroli et al., 2018).

Desde distintas disciplinas sanitarias ha sido percibido y definido como disfunción sexual. En este contexto, se ha abordado por diferentes saberes expertos a través de diversos tratamientos: Terapia dilatadora, asesoramiento y terapia sexual (Pacik, 2019), fisioterapia del suelo pélvico (Reissing et al., 2012) y recientemente, inyecciones de toxina botulínica (Karp et al,. 2019).

Así mismo, la mayor parte de investigaciones sobre vaginismo se han desarrollado desde disciplinas como la medicina, la psicología y la sexología, enfocadas principalmente en estudiar la prevalencia, la etiología y el tratamiento. Sin embargo, son escasas las investigaciones desarrolladas desde una perspectiva sociocultural que versen sobre vaginismo, u otras dificultades sexuales.

Partiendo de las bases teóricas anteriores, este artículo propone comprender las experiencias subjetivas de mujeres que han sido diagnosticadas de vaginismo a través del enfoque de la sociología de la sexualidad. El interés de la presente investigación radica principalmente en varias cuestiones. En primer lugar, el estudio de las dificultades sexuales a través de una perspectiva sociológica ha sido una temática escasamente abordada, ya que la sociología ha encontrado dificultades en el estudio de la sexualidad, al quedar reducida al dominio biomédico y psicológico. En segundo lugar, además de considerarse una dificultad sexual invisibilizada, apenas se han encontrado investigaciones que den voz a las mujeres con vaginismo a través de sus propias vivencias y experiencias subjetivas. Para dar respuesta a todas estas cuestiones, se ha optado por un enfoque cualitativo desarrollado a través de 8 entrevistas en profundidad.

Este artículo resulta pertinente, ya que ofrece un análisis alternativo sobre el vaginismo, otorgándole a la sociología de la sexualidad el espacio, a veces olvidado, del estudio de las dificultades sexuales.

\section{Marco teórico}

Las practicas sexuales han sido históricamente reguladas a través de normas de cortesía y etiqueta, otorgándole espacios, tiempos, modos y maneras (Guasch, 1993). La regulación de la actividad sexual ha pasado por distintas instituciones, desde la Iglesia y el cristianismo, creando una moral sexual férreamente procreadora, hasta el sistema biomédico, que se centra en remedios terapéuticos en busca de soluciones que se imponen hacia lo establecido como "normal" (Lucas 2009, Alcántara y Amuchástegui, 2009).

Según Atallah (2016), la normatividad sexual se manifiesta en forma de mensajes sociales y culturales, creando significado en las formas de comprender las dificultades sexuales e influyendo en las vivencias y las experiencias subjetivas de un sujeto con una disfunción sexual. 
En España, en concreto, el 52\% del total de la población piensa que el sexo de "verdad" incluye penetración y orgasmo. Por otro lado, el 59, 4\% piensa siempre, o casi siempre, durante las relaciones sexuales en la penetración como objetivo final de la actividad sexual (Ayuso y García, 2014). Esto no solo lo vemos en España, Szasz (1998) realiza una revisión de estudios sobre sexualidad en México y llega a la conclusión de que se le da gran importancia al coito y a la eyaculación, siendo las prácticas sexuales más deseadas. Jackson (1984) acuñó el término "imperativo del coito" para referirse a la idea socialmente construida que hace impensable tener relaciones sexuales sin penetración vaginal.

En esta línea, diversos autores apuntan que socialmente se tiende a equiparar relaciones sexuales con coito, y como consecuencia, el resto de las prácticas sexuales son obviadas o aceptadas como forma accesoria; provocando expectativas irreales, que a menudo tienen como consecuencia sentimientos de vergüenza, culpa y miedo (Andsager y Roe, 2003; Duran y Prusank, 1997; Kleinplatz et al., 2009; Krassas et al., 2001, 2003; Lucas, 2009; Ménard y Kleinplatz, 2008) Básicamente sigue prevaleciendo una sexualidad coitocéntrica, genitalizada y heteronormativa (Sanchis, 2009) y aquello que dificulta el coito es problematizado (Guasch, 1993).

Si el punto común de todos los autores es establecer que existe una sexualidad normativa, el sexólogo contemporáneo Lucas Matheu (2009) da un paso más, y añade que las consecuencias más visibles de esta normatividad sexual son las disfunciones sexuales, haciendo sufrir a una gran cantidad de personas, en muchos casos en silencio, produciendo que las relaciones sexuales se conviertan en encuentros caracterizados por la ansiedad de rendimiento, el miedo al fracaso y el límite del acceso al placer.

Ya Olga Viñuales (2002) apuntó que al tomar como referencia el coito vaginal en los encuentros sexuales, se limita la comprensión de la sexualidad y se patologiza todas las conductas que no se ajustan a este modelo, traduciéndose en el lenguaje como disfunción sexual, término psicológico y médico utilizado para describir o clasificar aquellas dificultades que imposibilitan el desarrollo de las prácticas sexuales consideradas principales y normativas.

De este modo, las dificultades sexuales comienzan a ser objeto a tratar por la medicina para responder a la supuesta sexualidad natural (Zavala \& Herrera, 2009).

Autoras como Cisternas y Niño (2014), van más allá, y conciben los tratamientos del vaginismo como vía para hacer los cuerpos de las mujeres funcionales, según los mandatos sociales y culturales; apoyándose en la idea foucaultiana que considera que lo definido como patológico en medicina, tiene sus causas en construcciones sociales de lo considerado "sexualidad natural" (Foucault, 1989).

Desde este enfoque, la perspectiva biomédica, pretende lograr que la sexualidad se sitúe entre los estándares de la "normalidad" (Alcántara y Amuchástegui, 2009).

A partir de esta lectura, lo funcional y lo disfuncional viene definido por características socioculturales de una sexualidad hegemónica, caracterizada por ser coitocéntrica y genital, definida y perpetuada por un sistema biomédico que establece distintos tratamientos en pro de hacer funcionales los cuerpos de las personas con dificultades sexuales.

\section{Metodología}

\subsection{Método}

El diseño de esta investigación se fundamenta en una metodología cualitativa de tipo exploratorio, en la que se ha utilizado entrevistas en profundidad como técnica de recopilación de datos. Se utilizaron entrevistas en profundidad ya que proporciona una gran riqueza informativa, al mismo tiempo que otorga privacidad y comodidad a las participantes, aspecto imprescindible cuando se tratan asuntos íntimos y personales. 


\section{1.a Participantes}

El número de entrevistadas fue de 8 mujeres heterosexuales que habían sido diagnosticadas de vaginismo y tratadas por diferentes expertos en distintos tratamientos.

Se han recogido, por tanto, narraciones centradas en las propias experiencias de mujeres con vaginismo. Los discursos de las participantes se encauzan principalmente en sus vidas afectivas y sexuales.

El diagnóstico, en concreto, fue de vaginismo primario para todas ellas, excepto para una participante, que fue diagnosticada de vaginismo secundario. La diferencia entre ambos es que, vaginismo primario se produce desde el inicio de las relaciones, mientras que el vaginismo secundario aparece después de haber podido mantener relaciones sexuales con penetración.

La edad de las participantes oscila entre los 20 y los 40 años. Las entrevistadas habían estado en diferentes tratamientos acompañadas de distintas profesionales: Sexólogas, psicólogas y fisioterapeutas del suelo pélvico.

Tabla 1

Característica de la muestra

\begin{tabular}{llll}
\hline & Tipo de vaginismo & Edad & Tratamiento \\
\hline 1 & Primario & 26 años & $\begin{array}{l}\text { Fisioterapia de suelo } \\
\text { pélvico }\end{array}$ \\
\hline 2 & Primario & 22 años & $\begin{array}{l}\text { Fisioterapia de suelo } \\
\text { pélvico }\end{array}$ \\
\hline 3 & Primario & 24 años & $\begin{array}{l}\text { Terapia psicológica } \\
\text { Primario }\end{array}$ \\
\hline 5 & 20 años & $\begin{array}{l}\text { Asesoramiento } \\
\text { terapia sexual }\end{array}$ \\
\hline 6 & Primario & 40 años & $\begin{array}{l}\text { Fisioterapia de suelo } \\
\text { pélvico }+ \text { Sexóloga }\end{array}$ \\
\hline 7 & Secundario & 29 años & $\begin{array}{l}\text { Fisioterapia de suelo } \\
\text { pélvico }+ \text { Sexóloga }\end{array}$ \\
\hline 8 & Primario & 22 años & $\begin{array}{l}\text { Asesoramiento } \\
\text { terapia sexual }\end{array}$ \\
\hline
\end{tabular}




\section{1.b Procedimiento y aspectos éticos}

El acceso a la muestra se realizó a través de distintos profesionales y otras mujeres que habían tenido vaginismo, mediante un muestro no probabilístico en forma de bola de nieve. Antes de cada entrevista, las participantes fueron informadas verbalmente y por escrito de los objetivos, procedimientos y propósitos de la investigación.

A todas las participantes del estudio se les hizo entrega del consentimiento informado y de un documento explicativo con las características metodológicas y éticas de la investigación, firmados por los investigadores. En ambos documentos se especifica el carácter voluntario de la investigación, el derecho a abandonar el estudio, a la confidencialidad y al anonimato.

Somos conscientes que el número de entrevistas no es muy alto, debido a que el acceso a la muestra estuvo limitado por las numerosas mujeres que rechazaron participar en la investigación, al tratarse aspectos tan íntimos y personales.

\section{1.c Análisis de los resultados}

Las entrevistas fueron grabadas y guardadas en archivos de audio para su posterior transcripción. El análisis del discurso fue temático y desarrollado a través del programa Atlas ti. Los pasos que se siguieron en el análisis del discurso fueron:

1. Transcripción y lectura de todas las entrevistas una vez finalizado el trabajo de campo.

2. A las participantes se les hizo entrega de las entrevistas, una vez transcritas, para asegurarnos que su discurso había sido reproducido correctamente

3. Se extrajeron unidades de significado, que fueron agrupadas en códigos.

4. Estos a su vez se agruparon en categorías y subcategorías, en función de la estructura informativa del discurso y del objetivo de la investigación

5. Se realizó una triangulación de datos con dos investigadores expertos en sexología

6. Finalmente, se procedió a desarrollar el análisis del discurso a partir de las categorías y las subcategorías derivadas del proceso de codificación.

\section{Resultados}

Del análisis de los discursos y narrativas de cada una de las participantes se pudieron extraer en concreto dos categorías principales, con sus respectivas subcategorías.

Tabla 2.

Categorías, subcategorías y códigos del análisis del discurso

\begin{tabular}{ccc}
\hline Categorías & Subcategorías & Códigos \\
\hline Vivir con vaginismo & Sentirse mujer fallida & $\begin{array}{c}\text { Nerviosa, dolor, no suficiente, } \\
\text { fallida, mujer incompleta, rabia, } \\
\text { tristeza, extrañas, raras, verguenza, } \\
\text { miedo, culpabilidad }\end{array}$ \\
\cline { 2 - 3 } & La experiencia con la pareja es \\
clave & $\begin{array}{c}\text { Compensar, culpabilidad, } \\
\text { afortunada, pacientes, presión, } \\
\text { chantaje, apoyo, fallar a la pareja, } \\
\text { miedo a la ruptura, injusto }\end{array}$ \\
\hline
\end{tabular}


Experiencias en las relaciones sexuales
Dolor, masturbación, estimulación, placer, sexo oral, caricias, relaciones incompletas, inseguras, evitar relaciones sexuales

El tratamiento del vaginismo

Ginecólogo, sexólogos, psicólogo,

Un camino complicado trabajo físico, trabajo emocional, educación sexual, autoestima, placer, solución, constancia, desconocimiento, invisibilidad

Relaciones sexuales tras el tratamiento
Nuevas formas de placer, dificultades, miedos, no placer con la penetración, evitar relaciones sexuales, normal.

\subsection{Vivir con vaginismo}

Las narraciones de las participantes están encauzadas en sus experiencias y vivencias afectivas y sexuales. El discurso se caracteriza por la asunción de la categoría de mujer disfuncional y la extrapolación de esta a sus relaciones sexuales y de parejas. Experiencias marcadas por creencias limitantes de la sexualidad, encuadradas dentro de un modelo normativo, caracterizado por ser coitocéntrico y genitalizado.

\section{1.a Sentirse mujer fallida}

El discurso de las mujeres con vaginismo está basado en gran parte en el dolor, pero no solo en el dolor físico, sino también emocional. En las narraciones mencionan el gran desconcierto que llegaron a sentir al no comprender qué les estaba sucediendo y al desconocer casos de otras mujeres que hubieran tenido una dificultad sexual similar.

"Ir a ciegas, no saber lo que me pasa, no saber cómo explicarlo, empezar por una parte y yo ya pensaba "Bueno, pues me tengo que hacer la idea de que yo nunca lo voy a poder hacer." (E2)

"Lo pasé muy muy mal, porque ves que es algo tuyo, que es tu culpa (...) te sientes que eres un bicho raro porque todo el mundo lo hace y todo el mundo se lo pasa tan bien y no entendía porque a mí me dolía tanto." (E6)

"Yo me sentía diferente al resto de las mujeres, no sabía qué pasaba y por qué yo no podía y para el resto como que todo era muy fácil. Cuando mis amigas hablaban de relaciones sexuales me sentía muy incómoda." (E5)

"Entonces yo sentía envidia y preocupación ¿Por qué a mí no me va bien y al resto sí? Porque claro, el resto de las respuestas, eran muy similares entre ellas, y mi experiencia pues era diferente, entonces era como muy frustrante." (E7)

Las narraciones se caracterizaban principalmente por la percepción centrada en la idea dicotómica de mujer normal vs mujer fallida. La mayor parte de ellas había asumido el rol de mujer fallida, que concurre con el hecho de no poder tener una relación sexual con penetración.

"Me ofuscaba mucho y me hacía sentir esto, que yo era menos, que era menos mujer, que estaba incompleta, que estaba mal hecha, algo fallaba en mí, que yo nunca podría dar lo que se tiene que dar, no soy una persona normal ¿Por qué no soy una persona normal? También es un ¿Por qué me está pasando esto a mí? ¿Por qué yo? "(E2) 
"Yo no era una mujer completa." (E3)

Al indagar sobre las ideas que habían influido en la forma de vivir y de percibir su dificultad sexual, se encontró que las vivencias estaban marcadas por ideas coitocéntricas y genitalizadas de la sexualidad. De hecho, las participantes hicieron referencia a que percibían, o habían llegado a percibir, que debía haber penetración para considerarse una práctica sexual completa, asumiendo la idea de que el resto de las prácticas sexuales eran preliminares, prácticas de segunda o relaciones sexuales no completas.

"Yo estaba completamente segura de que era lo principal (...) me obsesionaba con que si yo no podía me iban a dejar, que no iba a ser suficiente para mi pareja, que no estaba teniendo sexo." (E4)

Las participantes hicieron alusión a que normalmente la penetración solía considerarse implícita dentro de cualquier relación estable de pareja, sobre todo en un entorno donde creen que se les da una gran importancia a las relaciones sexuales.

"Todo el mundo asume que si tienes pareja tienes relaciones con penetración." (E2)

En algunos casos, estas ideas estaban plenamente presente en el entorno de las participantes, recibiendo mensajes de personas allegadas que las animaban a que tenía que aguantar el dolor si quería conseguir practicar por primera vez el coito. Una idea que prioriza la penetración, frente al placer sexual.

"Todas me decían que era muy extraño lo que me pasaba. Sobre la primera vez todas me decían que dolía mucho, y que tenía que aguantar, que tenía que hacer un esfuerzo, que yo me esforzara y que era una persona que no aguantaba el dolor, y que tenía que aguantar, soportar el dolor." (E3)

\section{1.b La experiencia con la pareja es clave}

Para las participantes resultó crucial las experiencias y vivencias dentro de la relación de pareja. En sus discursos resaltaron el miedo que sentían ante la posibilidad de que sus parejas decidieran abandonar la relación por no poder practicar el coito. Bajo estas ideas se fundamenta la creencia de que el placer de su pareja no iba a ser completo o suficiente sin penetración vaginal.

"Pero también está esa cosa de fallar a la pareja, de ser culpable de no poder dar algo que socialmente tiene que ir implícito en la relación ¿no? y de que quizás por culpa de eso pues me dejasen." (E6)

"La autoestima se me derrumbó, porque yo no me sentía suficiente, yo pensaba "Si no puedo, va a poder encontrarlo con otra chica y me va a dejar." (E4)

Pensaba que podían encontrar a alguien que podría dar algo que yo no podía (E8)

En base a esta idea, dos mujeres coincidían es sus discursos, afirmando que trataban de compensar el no poder practicar el coito, con otras características o cualidades. Intentar contrabalancear la imposibilidad de tener penetración con otros atributos, les produjo sentimientos de inseguridad en sus relaciones, percibiendo una disminución de su autoestima.

"Mi pensamiento era: Uno, no soy la suficientemente mujer porque no puedo darle lo que quiere, entonces su placer, nunca va a estar al 100\% conmigo, nunca va a estar al 100\%, por otra parte, tengo que compensar, tengo que ser super graciosa, super simpática, super perfectamente bella, para que él acepte que yo tengo esto." (E2)

\footnotetext{
${ }^{1}$ Haciendo referencia a la imposibilidad de practicar el coito.
}

Revista del Laboratorio Iberoamericano para el Estudio Sociohistórico de las Sexualidades https://doi.org/10.46661/relies.5295 
"Una cuestión de inseguridad, que hacía que yo no me relajara, y que yo me presionaba a mí misma, más la presión que tenía por su parte ¿sabes? (...) siempre me sentía como en una competición, y si no cumplo, sé que se van a ir." (E4)

Aun así, en las relaciones, encontramos parejas que supusieron un fuerte apoyo para las entrevistadas en relación con su vaginismo. Las parejas que sirvieron como apoyo, fueron descritas como pacientes, agradables y comprensibles. Las mujeres que han estado en relaciones empáticas manifestaron sentirse afortunadas. Sin embargo, todas estas mujeres, mantenían interiorizados sentimientos de culpa, por no poder practicar el coito, encontrándose intranquilas y preocupándose por la posibilidad de que sus parejas decidieran terminar con la relación.

"Yo con mi pareja puedo decir que he sido afortunada." (E5)

"Él era súper agradable, él nunca me hizo sentir intencionadamente al menos culpable, pero yo ya tenía interiorizado ese sentimiento, que yo ya lo sentía que era mi culpa ¿Sabes?" (E6)

Sin embargo, hubo excepciones, ya que seis de las participantes aludieron a que algunas de sus anteriores parejas (esporádicas y/o estables) las culpaban, presionaban y/o chantajeaban con tener prácticas sexuales con penetración, considerándose ellas mismas, en algunos momentos de su vida, como una mala novia/mujer.

"Me llegaban a decir cosas como de "Bueno pues me encantaría poder irme a dormir ahora, diciendo que rico que lo hemos pasado, pero como que no has querido..." como chantaje emocional." (E2)

"Lloraba, y a veces incluso lloraba del dolor, y a veces bueno obviamente parábamos y lloraba, y le pedía perdón. Le pedía perdón siempre. "Perdona porque no puedo. Él me lo hacía ver como si fuera una necesidad ¿me entiendes? Y si era una necesidad, yo decía, pues si no se lo puedo dar, que mala novia soy ¿sabes? me sentía horrible." (E4)

"Yo me sentía insegura y me sentía como que no cumplía." (E1)

Esta situación, fue ejemplificada con la historia de una de las participantes, que relató que su pareja alguna vez intentó practicar el coito mientras ella dormía. Incluso en su discurso alude a que llegó a entender la desesperación y la carencia de su pareja ante la necesidad de practicar el coito.

"Cuando yo dormía él intentaba la penetración, y en cuanto yo despertaba, me encontraba con eso y yo lloraba y después, él lloraba también, porque se sentía un monstruo y se sentía mal por estar haciendo eso ¿sabes? Y yo lo comprendía porque yo no lo conseguía, y él lo intentaba mucho y no lo conseguía." (E3)

\section{1.c Experiencia en las relaciones sexuales}

Cuando se indaga sobre otro tipo de prácticas sexuales, seis de las participantes manifestaron ser capaces de disfrutar del resto de prácticas, sin embargo, hicieron referencia a que llegaron a percibir que no tenían relaciones sexuales completas, clasificándolas, en algunos casos, como preliminares.

"Yo podía disfrutar con otro tipo de prácticas, para mí no tenía tanta importancia, pero me daba la sensación, y me la ha dado durante muchos años, que si no hay penetración no es placentero para el hombre." (E7)

Sin embargo, un par de participantes aludieron que debido a la culpabilidad y la inseguridad que les producía no poder tener relaciones con penetración, se consideraban incapaces de disfrutar del todo de otro tipo de prácticas sexuales.

"Digo la verdad nunca llegué a disfrutarlo $100 \%$ ese momento sí que estaba muy nerviosa, muy acomplejada porque te acompleja mucho. Todo, ya no estás tranquila, (...) en el que el momento se acerca, también estás un poco desconfiada, es decir, que no vaya a ser que les dé por intentarlo, ya tienes una asociación también como negativa, hacia todo eso." (E2)

Revista del Laboratorio Iberoamericano para el Estudio Sociohistórico de las Sexualidades https://doi.org/10.46661/relies.5295 
"Esta percepción de que tú no eres normal, y vivirlo mucho en secreto, es controlar mucho una relación sexual, y por lo tanto, yo creo que hubo momentos de mi vida en que he disfrutado mucho menos, porque claro como estás tan pensando, tan pendiente, todo es tan ¿no? tan pendiente, tanto pánico, te tan sientes expuesta. Yo me sentía expuesta en mis relaciones, frágil, vulnerable." (E6)

Se evidenció una clara tendencia y predisposición de las participantes a intentar tener relaciones sexuales con penetración a pesar del dolor. Indagando el por qué priorizaban el coito al placer y al no dolor, las respuestas fueron sentirse menos culpable, esforzarse para que la pareja no terminara con la relación, o en el caso de una de ellas, tener esta práctica porque la deseaba realmente.

"Me autoforzaba y no sé, no sé si me iba bien o mal, porque me esforzaba, me dolía, pero al menos se me quitaba un poco de culpabilidad." (E6)

"Yo intentaba, aunque me dolía mucho. Y muchas veces no quería porque estaba cansada y cosas así, pero yo lo intentaba siempre porque no quería que me dejasen." (E3)

Todas las participantes señalaron que los numerosos intentos fallidos de penetración, aumentaron sentimientos de inseguridad, miedo y culpabilidad, y en tres casos vieron deteriorado su deseo sexual o su capacidad erótica.

"Él no lo llevaba muy bien, porque quedábamos y ni siquiera lo intentábamos, por mi parte como que me quedaba bloqueada, y por su parte no quería hacerme daño. Me bloqueaba tanto que no conseguía hacer nada más. Era como que quería eso y quería eso, y no pensaba más." (E8)

"Cada vez se me fueron quitando las ganas de tener relaciones, yo no tenía ganas, porque ya pensaba en todo ese dolor, y además no solo es dolor físico, si no lo mal que lo pasaba yo porque me dolía psicológicamente." (E6)

\subsection{El tratamiento del vaginismo}

Los discursos de las entrevistadas hacen especial alusión al proceso de tratamiento. Todas las narraciones construyen un recorrido desde que empezaron a buscar una respuesta, pasando por el momento del diagnóstico, hasta su experiencia con el tratamiento y la repercusión de este en su vida sexual. Un camino marcado por la percepción de sentirse disfuncional con respecto a su cuerpo y a su sexualidad.

\section{2.a Un camino complicado}

Durante las entrevistas todas las participantes mencionaron la cantidad de tiempo que pasaron hasta conocer qué era lo que les sucedía, expresando que era debido a la invisibilidad que había alrededor del vaginismo, así como de cualquier aspecto de la sexualidad femenina. Todas ellas hicieron referencia a que la invisibilidad, el desconocimiento y la normalización del dolor por parte de los profesionales, fue la principal causa que hizo que el camino hasta encontrar un tratamiento adecuado destacara por ser complicado, arduo y difícil.

Curiosamente, la mitad de las participantes aludieron a que esta invisibilización y normalización del dolor, se encuentra dentro del mismo sistema sanitario, ejemplificando consultas ginecológicas, en las que curiosamente ninguna obtuvo información certera de lo que realmente acaecía. Estos profesionales expresaron que la cuestión era relajarse o que quizás no estaban lo suficientemente excitadas o lubricadas, aconsejándoles simplemente el uso de lubricante y, en uno de los casos, lubricante analgésico para poder practicar el coito.

"Le expliqué lo que me pasaba, la ginecóloga ni siquiera me miró, me dijo "Ah, pero tú eres virgen" y dije "si, pero porque no he podido" y me dijo "Nada, pues toma mucho lubricante y no ponerte nerviosa." (E2)

Revista del Laboratorio Iberoamericano para el Estudio Sociohistórico de las Sexualidades https://doi.org/10.46661/relies.5295 
En las narraciones se hizo alusión a sentimientos de rabia e impotencia por todo el dolor emocional que habían sufrido bajo el desconocimiento de lo que les ocurría. Sentimientos que afloraron especialmente al conocer el diagnóstico y la facilidad de llevar a cabo un tratamiento.

"Me puse a llorar a saco, o sea, sentía mucha rabia, había una parte de mí que pensaba "Vale, hay una respuesta, (...), y a la vez sentía como mucha impotencia ¿cómo me estoy enterando ahora?" (E1)

"Mantenía un sentimiento de culpa y de rabia sobre mí porque pensaba, madre mía, pero con lo fácil que es cómo que no he encontrado antes la solución, cómo nadie me había explicado nada." (E5)

Un sentimiento muy común en el momento del diagnóstico fue la ambivalencia entre felicidad por encontrar una respuesta y comprender qué les sucedía, y al mismo tiempo tristeza por tener que asumir la etiqueta de disfuncional y, por consiguiente, la necesidad de empezar un tratamiento. Bajo esta idea, los discursos oscilan en la patologización, la problematización del vaginismo y la percepción del cuerpo como fallido o disfuncional.

"Por una parte, es aceptar que tienes una condición ¿no? que tienes una afección, y es por una parte ser feliz porque sabes que tienes algo, y por otra parte es triste por saber que tienes algo, es decir, no son imaginaciones mías, tengo en realidad algo, estoy realmente enferma digamos, tengo realmente un problema." (E2)

A lo largo de la entrevista, hicieron hincapié en la decisión de acudir a un especialista a tratar su vaginismo. Destacaron que vivieron el tratamiento como un trabajo constante y duro, pero que realizaron con firmeza y esfuerzo con el objetivo principal de tener una relación sexual con penetración. Entre las profesionales que intervinieron con estas mujeres, hallamos expertas en psicología, sexología y fisioterapia del suelo pélvico ${ }^{2}$, tratamientos que se centraron en terapia física, educación sexual y autoconocimiento.

"Trabajamos conocerme, la autoestima, trabajamos con mi cuerpo, y creo que lo más me sirvió, fue cuando yo empecé con el vibrador, yo lo dejé, no volví a usarlo, empecé a experimentar con mi cuerpo, o sea yo sola a conocerme a mí." (E4)

"Se me hizo duro el tratamiento, estuve unas doce sesiones o así y claro, hacer como los deberes en casa, de hacer los ejercicios y tal, como lo tenía muy presente cada día, porque tener que hacer los ejercicios cada día y tal por un lado pensar "hazlo, porque, así como avanzas, progresas", pero a la vez no sé, también me costaba." (E1)

\section{$4.2 \mathrm{~b}$ Relaciones sexuales tras el tratamiento}

Al indagar sobre los resultados del tratamiento en las relaciones sexuales, dentro del discurso de las mujeres entrevistadas, se distinguen dos posiciones comunes que, en riesgo de reducir bastante, se pueden dicotomizar en, mujeres que se sienten capaces de disfrutar del coito después del tratamiento, y mujeres que no.

\footnotetext{
${ }^{2}$ Resulta interesante contemplar como todas las mujeres han estado acompañadas por otras mujeres profesionales y expertas en la temática. Las participantes habían acudido a estas profesionales casualmente, al descubrir su trabajo a través de recomendaciones, derivaciones o encontrarlas fortuitamente. Sin embargo, observar que todos las intervenciones y acompañamientos habían sido desarrollados por mujeres expertas, nos hacen cuestionarnos si el abordaje de la salud sexual femenina está desarrollado principalmente por mujeres profesionales o si existe alguna otra cuestión de género relevante que valorar. No se han encontrado investigaciones que dejen constancia del género de los/as expertos/as en el tratamiento del vaginismo y/o la dispareunia, sin embargo, sería interesante y revelador conocer las posibles cuestiones de género que afectan al perfil profesional de los tratamientos de la salud sexual femenina.
}

Revista del Laboratorio Iberoamericano para el Estudio

Sociohistórico de las Sexualidades 
Entre las entrevistadas que consideran que son capaces de disfrutar del coito, expresaron lo gratificante que es tener una nueva forma de dar y recibir placer, de estar relajada y de que la relación sexual pueda fluir. Pero en este mismo discurso, se evidencia una diferenciación, de nuevo, entre mujer normal y mujer fallida. Sin embargo, en este caso, justo después de tener relaciones placenteras con penetración, se consideran y acogen la etiqueta de "mujer normal"

"Me encuentro mejor conmigo misma en este sentido, me siento que tengo una vida normal, como en teoría debemos tener." (E6)

Sin embargo, todas las mujeres aludieron a que siguen sintiendo miedo o temor a que pueda manifestarse de nuevo la imposibilidad del coito, o de que la dificultad en la penetración aparezca en alguna relación sexual. Esto les provoca en algunos casos ciertas dificultades y límites en el acceso al placer, exponiendo características propias de lo que se conoce, en términos sexológicos, como ansiedad de ejecución ${ }^{3}$.

"Aún a veces me tengo que controlar, porque estoy nerviosa, me pesa, y digo que es mental sentirme como culpable ¿no? (...) es un proceso, que bueno, que sigo trabajando en ello (...) A veces tengo algún tipo de dificultad." (E6)

Por otro lado, hubo participantes que decían no haber podido experimentar aún una relación sexual plena y con penetración placentera, en la que pudieran desconectar del miedo a la imposibilidad o del temor a que pueda ser dolorosa.

En concreto, una entrevistada hace referencia a que sigue sintiendo dolor en la penetración y que era capaz de disfrutar más de sus relaciones sexuales antes del tratamiento. Declaraba que, una vez superado el vaginismo, y poseer la capacidad de practicar el coito, la penetración vaginal se consideraba necesaria e imprescindible en cualquier encuentro o relación sexual.

"Yo con mi expareja, que estuve un año, me lo pasaba mejor y disfrutaba mucho más cuando no sabía lo del vaginismo, y cuando empecé, a inicios del tratamiento, que al final. Porque claro, había una ley no escrita en la que a la que acabe el tratamiento, sí o sí tiene que haber esta relación con penetración y tiene que ser súper increíble, y fue una mierda." (E1)

Respecto a los discursos, hubo dos casos, en los que pudieron realizar un proceso de deconstrucción en el que trabajaron la idea de que una relación sexual sin coito puede ser muy placentera, y no es necesario para que un encuentro sexual sea completo. Sin embargo, lo miedos y las preocupaciones de tener dificultades en la penetración y/o no disfrutarla, siguen apareciendo cada vez que piensan en el momento de un nuevo encuentro sexual y/o de conocer a una nueva pareja.

"Cuando estoy en casa serena pienso, no se le tiene que dar tanta importancia, puede haber una relación sin penetración pero puede ser igual de guay, pero después yo que sé, ahora estoy conociendo a un chico con el que todavía no ha pasado nada, pero como me gusta y ya estoy pensando el día que me encuentre con el de repente en la cama, tiene que haber penetración, porque si no, como que parece que no está completo." (E1)

En un caso concreto, una mujer decidió evitar cualquier tipo de relación sexual, aunque lo deseara, ya que expresaba que siente pánico ante la situación de un encuentro sexual, que no lograba disfrutarlo y que le producía de nuevo un marco de ansiedad y frustración.

\footnotetext{
${ }^{3}$ La ansiedad de ejecución surge de experiencias fallidas que producen temor y pérdida de confianza ante el siguiente encuentro sexual. Se caracteriza por una enorme preocupación por conseguir un rendimiento óptimo y el sentimiento de miedo y temor por experimentar otra vez un nuevo fracaso. Esto les hace focalizar su atención en la evaluación de su comportamiento y función sexual, dificultando y/o limitando el acceso al placer en las relaciones sexuales (Navarro y Climent, 2013; Cymas y Crian, 2017)
}

Revista del Laboratorio Iberoamericano para el Estudio https://doi.org/10.46661/relies.5295

Sociohistórico de las Sexualidades 
"Tengo severo pánico ahora mismo porque es una situación que no me apetece nada, ni me lo paso bien, ni me renta, ni le renta a la otra persona (...) cuando estás ya tan nerviosa y has tenido todo este trauma detrás no estás sintiendo nada, no soy capaz de dejarme llevar y entonces yo lo he evitado desde entonces." (E2)

\section{Discusión}

Los resultados confirman que las mujeres participantes han interiorizado un modelo normativo de sexualidad, coitocéntrico, genitalizado y heteronormativo. Asumieron que la práctica sexual principal era la penetración, delegando el resto a preliminares o relaciones sexuales no completas. Estas ideas influyeron en la forma de comprender su dificultad sexual, viviendo sus experiencias avergonzadas, sintiéndose extrañas al resto de mujeres, sin entenderlo y en muchos casos en silencio.

Esto concuerda con el estudio de Bairstow (2018) en el que las mujeres con vaginismo participantes en su investigación sintieron vergüenza por no poder participar en algo que, comúnmente, se percibe como una actividad esencial y de no tener la capacidad de ejecutar una práctica que aparentemente el resto de las mujeres pueden desempeñar.

Atallah (2016) hace referencia a los factores relacionados con el papel social de la sexualidad, que influye en las expectativas de las relaciones sexuales. Creencias sociales, como la de que una mujer debe permitir relaciones sexuales coitales para complacer a su pareja, se traducen, en el caso de disfunciones sexuales, como el vaginismo o la dispareunia ${ }^{4}$, en sentimientos de ansiedad, culpa, responsabilidad por la falta de intimidad, de autonomía y control en las relaciones sexuales.

Ante la asunción de que la penetración es la práctica sexual principal, todas las entrevistadas se proyectaban como mujeres incompletas, fallidas y/o enfermas, concordando con los estudios de Farnam et al. (2014) y Stelko (2015), en los que las mujeres con vaginismo participantes de sus investigaciones reportaron percibirse menos mujer o mujer incompleta, además de sentirse frustradas y considerar que tenían un problema. Asimismo, estudios realizados sobre mujeres que padecen vulvodinia 5 , las participantes tomaron posiciones de "mujer inadecuada" cuando no podían participar regularmente en el coito (Ayiling, 2007). Tenemos que resaltar, además, que en sus discursos las mujeres destacan el sentimiento de sentir que su cuerpo está fallido y la asunción de la categoría de disfuncional.

Considerarse una mujer incompleta o fallida, produjo que una parte de las participantes de este estudio llegaran a disociar todas las prácticas sexuales del placer, por la inseguridad y la necesidad de control durante todo el encuentro sexual. Sin embargo, y en sintonía con el estudio de Cisternas (2015), las relaciones sexuales a veces se consideran placenteras, cuando el límite de la penetración estaba conversado con las parejas y la presión no tiene lugar, aunque siguen experimentando sentimientos de culpa y miedo al abandono. Aparentemente se debe a que consideraban otros tipos de prácticas como insuficientes para el placer de la pareja, no completas o preliminares. En vista de esto, las mujeres sienten que las parejas que no ejercen presión y culpabilidad son comprensivas y pacientes. Sáez (2009) caracteriza a las parejas de larga duración de las mujeres con vaginismo, como comprensivos y respetuosos, adjetivos que las participantes de este estudio han dado

\footnotetext{
${ }^{4}$ La dispareunia es una dificultad sexual, identificada en el DSM-V como trastorno genito-pélvico de dolor/penetración (GPPPD), y se caracteriza por la aparición de dolor genital antes, después o durante una relación sexual con penetración. A diferencia del vaginismo, el coito no es imposible, pero si doloroso.

${ }^{5}$ Vulvodinia es el "malestar vulvar, más a menudo descrito como dolor ardiente, que ocurre en la ausencia de hallazgos visibles relevantes o trastornos neurológicos específico, clínicamente identificable." (Haefner et al., 2005, pags. 41).
} 
también a sus propias parejas. Sin embargo, al igual que en el estudio de Cisternas (2015) las mujeres también aluden a parejas que las presionaron y culparon por no tener relaciones coitales.

Se observa una clara disposición de todas las participantes a intentar persistentemente el coito, ante el temor del abandono de la pareja o para que el sentimiento de culpa disminuyera. En concordancia con la investigación de Cisterna (2015), la autora señala que el dolor en la penetración es legitimado dentro de un modelo de sexualidad que sólo tiene justificación en el sacrificio corporal de la mujer. Siguiendo esta línea, Cisternas (2015), expone que la mujer sigue sometiéndose al dolor para responder a un coito normativo, dónde, según Irigaray (2010) la función de la vagina se presenta principalmente como receptáculo del pene.

Sin embargo, tres de ellas en algunos momentos de su vida, terminaron por evitar cualquier tipo de práctica sexual, ya que cada intento fallido, las frustraba aún más y bloqueaba otras expresiones sexuales, situación acentuada por lo sentimientos de culpa y vergüenza.

Podemos ver un reflejo de estos resultados en el estudio de Dias y Marques (2018) y Fadul et al., (2018) donde las mujeres con vaginismo mostraron una mayor evitación a las relaciones sexuales. La vergüenza puede desembocar al silenciamiento y aislamiento (Seu, 1995). Según Ayling (2008) las mujeres de su estudio sobre vulvodinia, que adoptan una posición de "mujer / pareja sexual inadecuada" podían ver los actos sexuales no penetrativos, como un recordatorio de su incapacidad para satisfacer a su pareja sexualmente. Esta situación provoca culpa y un menor deseo de contacto sexual, acentuado por el miedo al dolor físico y el silencio causado por la vergüenza.

Sin embargo, las participantes de nuestro estudio no solo muestran miedo al dolor físico, sino también al fuerte dolor emocional producido por los intentos de penetración fallidos, la inseguridad y la frustración por no poder practicar el coito.

Las participantes buscaron a través de diferentes recursos la solución, expresando la dificultad que tiene encontrarla ante la invisibilización, el silencio que rodea al vaginismo y el desconocimiento de algunos profesionales. Esto concuerda con los estudios de Macey et al. (2015) e Ishaq y Rehman (2014) en los que las participantes demostraron sentirse tímidas a buscar ayuda. Sin embargo, son los sentimientos de vergüenza, anormalidad y el desconocimiento de muchos profesionales de la salud, la principal barrera que dificulta conocer que les ocurre. Así parece mostrarlo este estudio, y muchos otros, en los que las mujeres se sintieron mal atendidas, y en los que los profesionales les aconsejaron utilizar lubricante o juegos previos al coito, sin dar una respuesta real a lo que les sucedía (Macey et al., 2015).

Cuando descubrieron que tenían vaginismo, todas recurrieron a profesionales y expertas para empezar un tratamiento. La mayoría de ellas fueron tratadas principalmente a través de la dilatación progresiva, concordando con los estudios de Stelko (2015) y Cisterna (2015), donde la prevalencia de tratamiento basado en la dilatación era la más frecuente.

En concordancia con estudios anteriores, las mujeres que acudieron a fisioterapia de suelo pélvico calificaron este tratamiento de forma más positiva, destacando su utilidad y satisfacción (Reissing et al., 2003 y Reissing, 2012)

Una vez superado el vaginismo, dando el tratamiento como finalizado, una parte son capaces de disfrutar del coito, sin embargo, siguen sintiéndose inseguras y temerosas ante un nuevo fracaso, provocando en algunos momentos dificultades en la penetración y limites en el acceso al placer. En estos casos, se presenta características propias de la ansiedad de ejecución.

Otras mujeres, ante la imposibilidad de disfrutar de la penetración, han realizado un trabajo de deconstrucción en base a la idea de que una relación sexual puede ser completa y muy placentera sin coito. Aun así, siguen sintiéndose inseguras ante un nuevo encuentro sexual, por miedo a ser rechazadas.

Revista del Laboratorio Iberoamericano para el Estudio https://doi.org/10.46661/relies.5295

Sociohistórico de las Sexualidades 
Una de las entrevistadas ante la incapacidad de disfrutar del coito, y del resto de relaciones sexuales por las inseguridades y complejos que le causó haber tenido vaginismo, decidió evitar cualquier tipo de encuentro sexual.

El estudio de Reissing et al., (2003) reporta que las mujeres con vaginismo entrevistadas indicaron que obtuvieron la capacidad de disfrutar de la penetración como resultado del tratamiento de la fisioterapia, pero que algunos síntomas de dolor, tensión del suelo pélvico, ansiedad y miedo permanecieron.

En nuestra investigación, no todas las mujeres obtuvieron la capacidad de disfrutar del coito, pero todas siguieron manteniendo cierta dificultad, ansiedad o miedo, algunas de ellas incluso dolor o apareciendo a veces la imposibilidad de penetración, independientemente del profesional que llevó a cabo el tratamiento.

Por otro lado, es interesante destacar, que en los casos en que las participantes llegaron a construir la idea de que la penetración es una actividad sexual más, siguen refiriéndose al resto de prácticas sexuales como preliminares, otorgándole al coito una categoría superior dentro del encuentro sexual.

Las participantes asumen la categoría de disfuncionales, incluso aquellas que han realizado el mencionado trabajo de deconstrucción. En muchos casos se suelen referir a su situación como problemática o no saludable. Por lo tanto, sus discursos no sólo muestran el sentimiento de ser una mujer fallida, si no también, la percepción del cuerpo fallido. Bajo esta idea, los discursos oscilan en la patologización, la problematización del vaginismo y la asunción de la categoría de disfuncional. El cuerpo, y las funciones atribuidas a él, son centrales en la construcción del género, por lo tanto, se aprecia como disfuncional en la medida que no permite realizar ciertas funciones atribuidas a la femineidad.

En este estudio se pone de manifiesto cómo el tratamiento se dio por finalizado cuando se permitió un coito indoloro, o cuando se concluyó con una dilatación suficiente para la introducción de un pene. El tratamiento ha estado centrado en hacer funcionales los cuerpos de las mujeres, en base al modelo normativo de sexualidad, coitocéntrico y genitalizado. Sin embargo, el placer al integrar el coito en su actividad sexual no se ha atendido suficientemente por los profesionales, produciéndose en algunos casos ansiedad de ejecución, el no disfrute de las relaciones sexuales o la evitación de la actividad sexual.

Un enfoque interdisciplinar de las intervenciones podría concluir en la deconstrucción de los discursos e ideas interiorizadas de una sexualidad normativa, con el fin de desafiar el impacto significativo que tiene en la sexualidad de las mujeres con vaginismo. Cuando se incluye la penetración como una práctica más y se deja de considerar el coito como esencial, se permite la exploración de prácticas alternativas, permitiendo reducir o abandonar sentimiento de culpabilidad o de ansiedad ante dificultades en la penetración.

\section{Conclusiones}

Los resultados muestran cómo las mujeres con vaginismo han interiorizado ideas de una sexualidad normativa, influyendo en sus experiencias subjetivas con relación a su dificultad sexual.

Las historias de las mujeres entrevistadas se enmarcan en un contexto social heteronormativo, dónde el coito se presenta como la práctica sexual normativa y principal. Es curioso, como en sus descripciones se refieren al fallo de su cuerpo y género, definiéndose como mujer fallida o mujer incompleta. La imposibilidad de penetración se muestra cómo el impedimento para participar en una actividad considerada esencial para el desempeño de su género, y así se representa en sus discursos, mediante las declaraciones del deseo de ser una "mujer normal". 
En relación con esto, varias mujeres han sentido que al no poder realizar una práctica sexual que se considera implícita dentro de la heteronormatividad, el placer de sus parejas iba a estar incompleto. Han buscado soluciones al vaginismo, en la mayoría de los casos, para adaptarse a las prácticas sexuales normativas que se han definido dentro de este modelo.

Así mismo, el tratamiento del vaginismo se ha reducido a un plano donde las intervenciones se han sido desarrolladas desde la perspectiva de lo patológico. Los tratamientos se han llevado a cabo con el fin de permitir el coito, y hacer normativos los cuerpos de las mujeres, sin prestarle atención a la incorporación del coito como práctica placentera y a la deconstrucción de este modelo que limita el disfrute en las relaciones sexuales.

Dentro de este punto, es interesante hacer referencia a la alusión del cuerpo como fallido o disfuncional, tanto en la apreciación de ciertos discursos profesionales, hasta la propia percepción de las entrevistadas. Principalmente, el cuerpo se aprecia como disfuncional en la medida que no permite realizar ciertas funciones atribuidas a la feminidad.

En general, esta investigación evoca la importancia que adquiere el coito en la forma de vivir el género, el cuerpo y la sexualidad, así como la compleja interrelación entre cuerpo, género y prácticas sexuales.

Al ser una investigación cualitativa, no se busca realizar ninguna inferencia, sin embargo, el relato de las experiencias subjetivas de diferentes mujeres que han padecido vaginismo nos acerca a su realidad, contribuyendo al planteamiento de nuevas líneas de intervención.

Este estudio presenta la necesidad de un abordaje dirigido a despatologizar el vaginismo y a deconstruir los discursos normativos de la sexualidad, con la intención de que el coito deje de considerarse esencial, permitiendo priorizar prácticas alternativas de placer. Una intervención que cuente con el objetivo de evitar en las mujeres con vaginismo la presión, minimizando los sentimientos culpa, frustración y miedo, evitando la percepción y posicionamiento del cuerpo como "disfuncional".

Sería interesante como nueva línea de investigación, ampliar la muestra para incluir a mujeres que deciden no acudir a ningún profesional y/o mujeres con distintas orientaciones sexuales. Probablemente nos dé una visión más completa y global de cómo se vive este fenómeno socialmente. 


\section{Bibliografía}

American Psychiatric Association (APA). (2013). Manual Diagnóstico y Estadístico de los Trastornos Mentales DSM-V-TR. Barcelona: Masson

Alcántara Z., Eva y Amuchástegui H., Ana. (2009). Terapia sexual y normalización: Significados del malestar sexual en mujeres y hombres diagnosticados con disfunción sexual. Physis, 19(3): 591-61.

Andsager, Julie y Roe, Kimberly. 2003. What's your definition of dirty, baby?: Sex in music video" Sexuality \& Culture, 7:79-97.

Atallah, Sandrine et al. (2016). Ethical and Sociocultural Aspects of Sexual Function and Dysfunction in Both Sexes. Journal of Sexual Medicine, 13(4): 591-606. DOI: 10.1016/j.jsxm.2016.01.021.

Ayling, Kathryn y Ussher, Jane M. (2008). "If sex hurts, am I still a woman?" The subjective experience of vulvodynia in hetero-sexual women". Archives of Sexual Behavior, 37(2): 294-304. DOI: 10.1007/s10508-007-9204-1.

Ayuso, Luis y García, Livia. (2014). Los españoles y la sexualidad en el siglo XXI. Monografía Centro de Investigaciones Sociológicas, 281.

Bairstow, Adrienne M. et al. (2018) Holy Grail or nice option: The meaning of penile-vaginal Intercourse in "unconsummated" relationships. The Canadian Journal of Human Sexuality, 27(2): 19. DOI:10.3138/cjhs.2018-0015.

Cimas, Magali y Cryan Glenda (2017). Análisis del consumo de potenciadores sexuales en jóvenes y adultos mayores. IX Congreso Internacional de Investigación y Práctica Profesional en Psicología XXIV. Jornadas de Investigación XIII Encuentro de Investigadores en Psicología del MERCOSUR. Facultad de Psicología. Universidad de Buenos Aires, Buenos Aires.

Cisternas, Manuela y Niño, Elisa (2014). Mandatos sexuales y vaginas inteligentes. Aproximación desde la narrativa de mujeres con vaginismo. III Congreso de Género y Sociedad "Voces, cuerpos y derechos en disputas.

http://conferencias.unc.edu.ar/index.php/gyc/3gyc/paper/view/2635/710, último acceso 22 de Mayo de 2020.

Cisternas, Manuela (2015) Sexualidad y cuerpos en relatos de mujeres con vaginismo. Universidad de Chile. (En línea) http://repositorio.uchile.cl/handle/2250/142338, último acceso 22 de Mayo de 2020.

Dias A., Ana y Marques P., André (2018). Female Genito-Pelvic Pain / Penetration Disorder : Review of the Related Factors and Overall Approach. Revista Brasileira de Ginecologia e Obstetrícia, 40(12): 787-793 DOI: 10.1055/s-0038-1675805.

Duran, Robert L., \& Prusank, Diane T. (1997). Relational themes in men's and women's popular nonfiction magazine articles. Journal of Social and Personal Relationships, 14 (2) 165-189. 
Fadul Rosario et al. (2018). Psychosocial correlates of vaginismus diagnosis: A case-control study. Journal of Sex \& Marital Therapy, 45(1): 73-83. DOI: 10.1080/0092623X.2018.1484401.

Farnam, Farnaz. (2014). Vaginismus and its correlates in an Iranian clinical sample. International Journal of Impotence Research, 26(6): 230-234. DOI: 10.1038/ijir.2014.16.

Foucault, Michael. (1989). La vida de los hombres infames. Ensayos sobre desviación y dominación. España: La Piqueta.

Guasch, O. (1993). Para una sociología de la sexualidad. Reis: Revista Española de Investigaciones Sociológicas, 64 (93): 105-122. DOI: 10.1177/1461444814521595.

Haefner, H., Collins, M. E., Davis, G. D., Edwards, L., Kaufman, R. H., Lynch, P. J., et al. (2005). The vulvodynia guide. Journal of Lower Genital Tract Disease, 9, 40-51.

Irigaray, L. (2010). Ética de la diferencia sexual. Ellago Ediciones.

Ishaq, Humera y Rehman, Hina (2014). Vaginismus: Cross-sectional study in population of Karachi, Pakistan. International Journal of Pharm and Drug Analysis, 2(11): 849-857. ISSN: 2348-8948.

Jackson, Margaret (1984). Sex research and the construction of sexuality: A tool of male supremacy? Women's Studies International Forum ,7(1): 43-51.

Karp, Barbara (2019). Methodological approaches to botulinum toxin for the treatment of chronic pelvic pain, vaginismus, and vulvar pain disorders. International Urogynecology Journal, 30(7): 10711081. DOI: 10.1007/s00192-018-3831-z.

Kleinplatz, Peggy J. et al. (2009). The components of optimal sexuality: A portrait of great sex. The Canadian Journal of Human Sexuality, 18(1): 1-13.

Krassas, Nicole R., Blauwkamp, Joan M., \& Wesselink, Peggy (2001) Boxing Helena and corseting Eunice: Sexual rhetoric in Cosmopolitan and Playboy magazines. Sex Roles, 44(11): 751-771. DOI: 10.1023/A:1012254515434.

Krassas, Nicole R., Blauwkamp, Joan M., \& Wesselink, Peggy (2003). Master your Johnson: Sexual rhetoric in Maxim and Stuff magazines. Sexuality and Culture: An Interdisciplinary Quarterly, 7(3):98119.

Lucas M., Manuel (2009) Sed de piel ¿Feminizar el futuro? Madrid: Psimática

Macey, Kat et al. (2015). Women's experiences of using vaginal trainers (dilators) to treat vaginal penetration difficulties diagnosed as vaginismus: A qualitative interview study. BMC Women's Health, 15 (49): 1-12. DOI: 10.1186/s12905-015-0201-6.

Maseroli, Elisa et al. (2018). Outcome of Medical and Psychosexual Interventions for Vaginismus: A Systematic Review and Meta-Analysis. Journal of Sexual Medicine, 15(12): 1752-1764. DOI: 10.1016/j.jsxm.2018.10.003.

Ménard A., Dana., y Kleinplatz, Peggy J. (2008). 21 moves guaranteed to make his thighs go up in flames: Depictions of "great sex" in popular magazines. Sexuality \& Culture, 12(1): 1-20.

Revista del Laboratorio Iberoamericano para el Estudio Sociohistórico de las Sexualidades https://doi.org/10.46661/relies.5295 
Moltedo-Perfetti, Andrés; Nardi, Bernardo y Arimatea, Emilio (2014). Coherencia sistémica e identitaria en mujeres con vaginismo primario. Revista Chilena de Obstetricia y Ginecología, 79(1): 56-63. DOI: 10.4067/S0717-75262014000100010.

Navarro A, Yolanda y Climent R, José A (2013). Tratamiento cognitivo-conductual de un caso de trastorno de la erección adquirido. Medicina psicosomática y psiquiátrica. Revista Iberoamericana de psicosomática, 105, 72-79.

Osborne, Raquel y Guasch, Oscar. (2003). Sociología de la Sexualidad. Revista Internacional de Sociología, 38, 221-224.

Pacik, Peter T. (2014). Understanding and treating vaginismus: a multimodal approach. International Urogynecology Journal and Pelvic Floor Dysfunction, 25(12): 1613-1620. DOI: 10.1007/s00192-0142421-y.

Pacik, Peter T. (2019). Case Series: Redefining Severe Grade 5 Vaginismus. Sexual Medicine, 7 (4): 489-497. DOI: 10.1016 / j.esxm.2019.07.006.

Reissing, Elke D. et al. (2003). Etiological Correlates of Vaginismus: Sexual and Physical Abuse, Sexual Knowledge, Sexual Self-Schema, and Relationship Adjustment". Journal of Sex \& Marital Therapy, 29(1): 47-59. DOI: 10.1080/713847095.

Reissing, Elke D. (2012). Consultation and treatment history and causal attributions in an online sample of women with lifelong and acquired vaginismus. The Journal of Sexual Medicine, 9(1): 251258. DOI: 10.1111/j.1743-6109.2011.02534.x.

Sánchez Bravo, C., Carreño Meléndez, J., Corres Ayala, N. P., \& Henales Almaraz, C. (2010). Perfiles e indicadores psicológicos relacionados con la dispareunia y el vaginismo. Estudio cuantitativo. Primera parte TT". Salud Mental, 33(4), 347-353. Disponible en:http://www.scielo.org.mx/scielo.php?script=sci\%7B \%7Darttext\%7B\&\%7Dpid=S01833252010000400007\%7B\&\%7Dlang=pt\%5Cnhttp://www.scielo.org.mx/pdf/sm/v33n4/v33n4a7.pd f.

Sanchis, Rosa (2009). Machitos, supernenas y demás fauna adolescente ¿Y quién no tiene las hormonas revolucionadas?. Jornadas feministas Granada, (en línea) http://www.rosasanchis.cat/articles_ponencies.html, último acceso 22 de Mayo de 2020.

Sáez S., S. (2009). El Vaginismo y su dialéctica sexuada". Anuario de Sexología, 11(1): 27-33. 